Article

\title{
Predesign Considerations for the DC Link Voltage Level of the CENTRELINE Fuselage Fan Drive Unit
}

\author{
Stefan Biser ${ }^{1,2, *,+(\mathbb{D} \text {, Guido Wortmann }}{ }^{1,+}{ }^{\dagger}$ Swen Ruppert ${ }^{3, \dagger}{ }^{+}$, Mykhaylo Filipenko ${ }^{1, \dagger}{ }^{\dagger}$, \\ Mathias Noe ${ }^{2}$ and Martin Boll ${ }^{1,+}+\mathbb{D}$ \\ 1 Rolls-Royce Limited \& Co. KG, Willy-Messerschmitt-Str. 1, 82024 Taufkirchen, Germany; \\ guido.wortmann@rolls-royce.com (G.W.); mykhaylo.filipenko@rolls-royce.com (M.F.); \\ martin.boll@rolls-royce.com (M.B.) \\ 2 Karlsruhe Institute of Technology-Institute of Technical Physics, Hermann-von-Helmholtz-Platz 1, \\ 76344 Leopoldshafen-Eggenstein, Germany; mathias.noe@kit.edu \\ 3 Siemens AG, Anton-Bruckner-Str. 2, 91052 Erlangen, Germany; swenruppert@t-online.de \\ * Correspondence: stefan.biser@rolls-royce.com \\ + These authors contributed equally to this work.
}

Received: 2 October 2019; Accepted: 14 November 2019; Published: 20 November 2019

\begin{abstract}
Electric propulsion (EP) systems offer considerably more degrees of freedom (DOFs) within the design process of aircraft compared to conventional aircraft engines. This requires large, computationally expensive design space explorations (DSE) with coupled models of the single components to incorporate interdependencies during optimization. The purpose of this paper is to exemplarily study these interdependencies of system key performance parameters (KPIs), e.g., system mass and efficiency, for a varying DC link voltage level of the power transmission system considering the example of the propulsion system of the CENTRELINE project, including an electric motor, a DC / AC inverter, and the DC power transmission cables. Each component is described by a physically derived, analytical model linking specific subdomains, e.g., electromagnetics, structural mechanics and thermal analysis, which are used for a coupled system model. This approach strongly enhances model accuracy and simultaneously keeps the computational effort at a low level. The results of the DSE reveal that the system KPIs improve for higher DC link voltage despite slightly inferior performance of motor and inverter as the mass of the DC power transmission cable has a major share for a an aircraft of the size as in the CENTRELINE project. Modeling of further components and implementation of optimization strategies will be part of future work.
\end{abstract}

Keywords: electric propulsion; aircraft; CENTRELINE; DC link voltage level; analytical model; design space exploration

\section{Introduction}

While noise levels have been reduced drastically, absolute emissions from aviation, especially carbon dioxide $\left(\mathrm{CO}_{2}\right)$ and nitrogen oxide $\left(\mathrm{NO}_{x}\right)$, have been continuously on the rise throughout the last decades, accounting for 2-3\% of global greenhouse gas emissions. The large improvements in fuel efficiency are thwarted by the steady increase in passenger numbers [1,2]. The National Aeronautics and Space Administration (NASA) N+3 fundamental technology research for subsonic fixed-wing aircraft [3,4], the Civil Aviation Organization (ICAO) [5,6] as well as the Aviation Transport Action Group (ATAG) [2] and the European Commission's Flightpath 2050 [7] set aggressive target levels for greenhouse gas emissions in the future-up to 75\% less fuel burn and 90\% NOx emissions by 2050.

To reach those ambitious objectives, a significant improvement in terms of fuel consumption is necessary. These objects can be seen as one of the main drivers for the development of novel 
propulsion systems [8], resulting in a large increase in interest on partly or fully electrified aircraft $[9,10]$. The change to electric-based propulsion systems not only affects current designs, it completely changes the setup. Three types of EP for aircraft can be distinguished by their level of hybridization [9]. Turbo-electric propulsion systems have a total hybridization of power, i.e., they generate all power from burning fuel in gas turbines [11,12]. Hybrid-electric concepts involve power generation from burning fuel and electric storage [8,13]. All-electric propulsion systems only have batteries on-board for energy storage [14].

An often-discussed point is the choice of the DC link voltage level for power transmission. The voltage level of state-of-the-art aircraft is currently limited to $\pm 270 \mathrm{~V} D C$ to avoid corona at typical flight altitudes $[15,16]$. Increasing electric power demand of aircraft requires higher voltage levels to reduce weight and keep transmission efficiency at a high level [14,16,17]. Considering DC link voltages from $1000 \mathrm{~V}$ to over $5000 \mathrm{~V}$, several studies showed that the mass of the power transmission cable resulting from the choice of the DC link voltage level is an important driver in system mass [15,17]. Up to now, the sensitivity of the electric machine and inverter design for electric aircraft on the DC link voltage has not yet been investigated in a coupled research.

In this paper, we elaborate this problem, having a closer look on the propulsion system of the CENTRELINE project [18] where $8 \mathrm{MW}$ of propulsion power are required. In contrast to conventional aircraft powered by gas turbines, especially turbo-electric and hybrid-electric propulsion systems have significantly increased complexity on the system level and mass. Due to the increased number of components with a large number of parameters influencing the mission performance, a large design space is opened up [9,12]. Brelje and Martins [9] reviewed many approaches which try to incorporate sizing methods for EP on different levels of detail into the conventional aircraft sizing procedure. However, most of them use simple correlations based on stochastic regression or fixed parameters for specific power and efficiency of the components within the electric drivetrain [8,12,19-23]. Accordingly, EP is still poorly understood due to the low fidelity models that are used [9]. Thus, they proposed to develop a fully coupled, multidisciplinary analysis and optimization (MDAO) for electric aircraft.

As such models require enormous calculation power and long calculation time, we follow a different approach in this paper where each component is described by a physically derived, analytical model linking several subdomain models specific to the component together; e.g., for an electric machine, these are electromagnetics, structural mechanics and thermal analysis. All such components models are finally set up as coupled model for the complete system. This approach aims to strongly enhance the prediction accuracy, especially in terms of plausibility, reliability, and traceability of the results, while at the same time keeping the computational effort low to perform large design space explorations.

The paper is structured as follows: Firstly, the CENTRELINE project and the reference propulsion architecture are shortly introduced. Secondly, the specific challenge is presented, and the sizing process of the components is explained in more detail. The results of the large design space exploration are discussed component-wise as well as for the combined system. After a discussion of the results, an outlook of future work will be given.

\section{CENTRELINE Project Overview}

CENTRELINE is a project funded by the European Union (EU), serving as a demonstrator for propulsion-aircraft integration with the goal of validating results of former studies on boundary layer ingestion (BLI) such as the FP7 project DisPURSAL [24]. The major change in aircraft design is the implementation of the so-called fuselage fan (FF) drive unit which will be installed in the fuselage aft-end [18]. This device ingests and re-energizes the boundary layer of the fuselage to compensate viscous drag in the fuselage boundary layer flow $[18,25]$.

While DisPURSAL [24] focussed on a conventionally driven fuselage propulsor by a third gas turbine, CENTRELINE will evaluate the possible advantages of a fully turbo-electric drivetrain arrangement to overcome shortcomings of a gas turbine in the aft section such as flow losses associated 
with the air intake duct, vibration issues and high maintenance costs [26]. The electric powertrain consists of two power generation sets, an electric power transmission system as well as a single fuselage propulsor located in the rear of the aircraft, as shown in Figure 1. Each generation is composed of a wing-podded, advanced geared turbofan (GTF), an electric generator that converts mechanical to electric power, and an AC/DC rectifier. The electric generator is mechanically coupled to an additional free power turbine located in the low-pressure turbine (LPT) section of the GTF even though this is in the hot section, as this has the minimal influence on engine stability. The rectifier will be placed in the nacelle of the GTF to evaluate the potential of air-cooled power electronics [23].

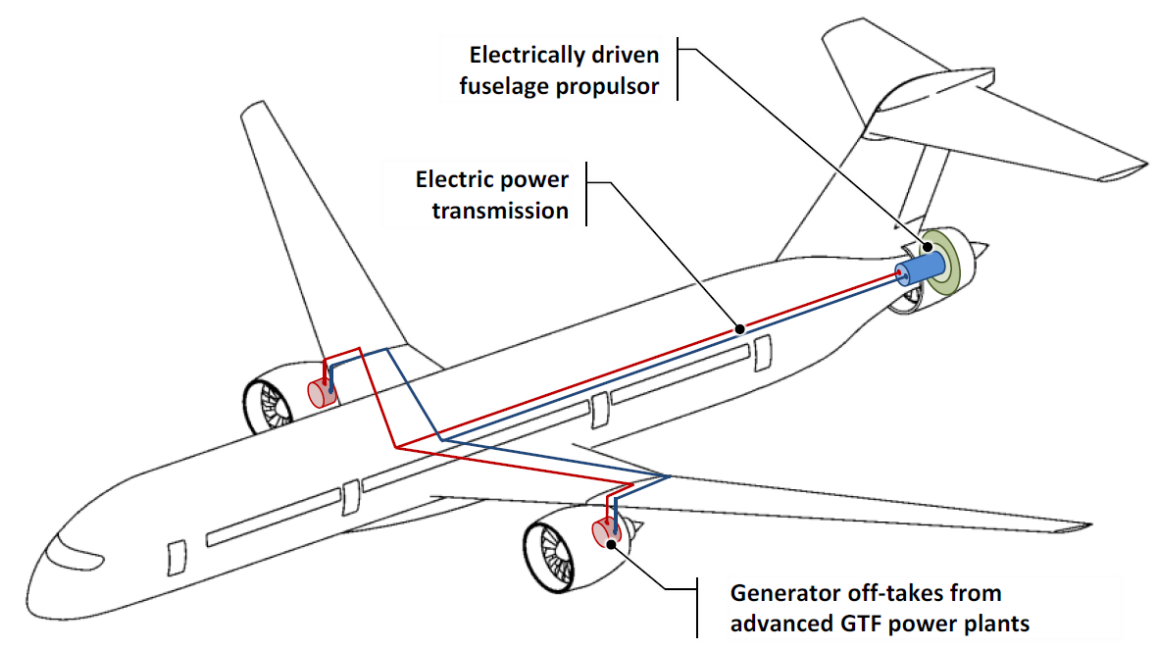

Figure 1. H2020 CENTRELINE architecture (turbo-electrically driven fuselage wake-filling) [18].

A DC power transmission system is chosen, consisting of eight parallel lanes to transfer the power as well as protection and switching devices to control it. In the rear, a single propulsion unit including eight parallel DC / AC inverters, one electric motor and the fuselage fan itself is mounted. The inverter converts electric DC to AC power, while the motor converts it to mechanical power to drive the fuselage fan directly without any additional gearbox [27].

Several limitations with regards to installation space of inverter and electric motor (depicted in Figure 2), derived by aerodynamic calculations, must be considered during the sizing process.

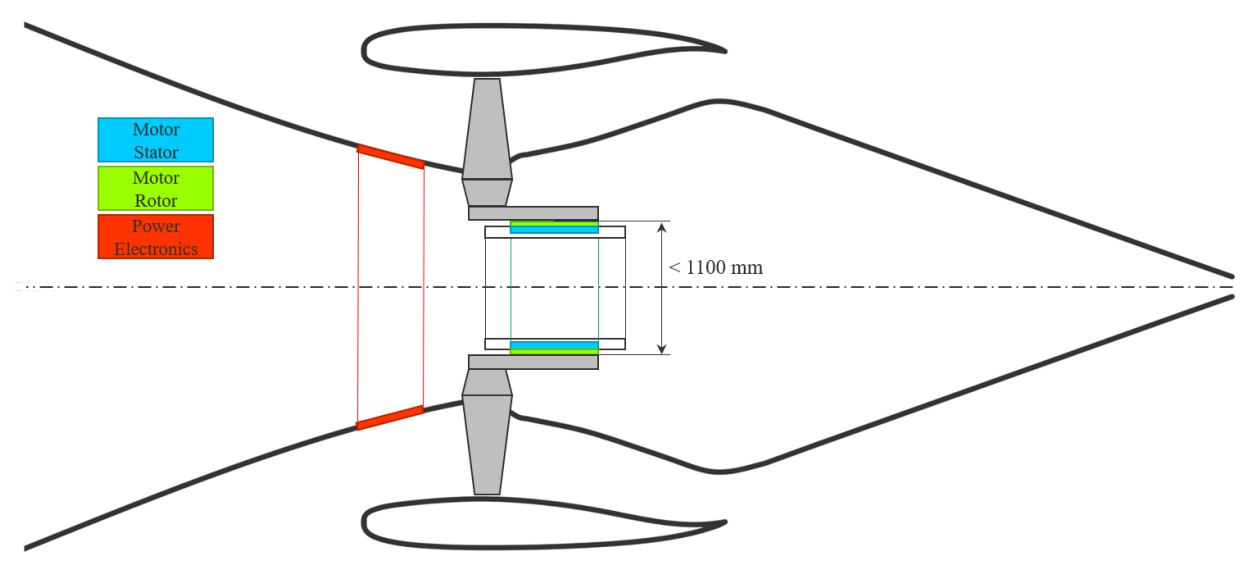

Figure 2. Fuselage fan drive unit integration with installation space limitations (taken from [27] and modified). 


\section{Methodolgy}

\subsection{Problem Setup}

Brelje and Martins [9] as well as Gesell et al. [12] stated that EP is not applicable as drop-in replacement of existing aircraft propulsion unit but has to be optimized for aircraft and mission requirements. As outlined in Section 1, the proposed approach of sizing components of the electric drivetrain with physically derived, analytical models systematically improves the level of model details in order to raise awareness of importance of a better understanding of EP systems for aircraft. The average run-time per system design point is within the small digits of seconds on an Intel ${ }^{\mathbb{R}}$ Core $^{\mathrm{TM}}$ i7-6600U CPU @ $2.60 \mathrm{GHz}$.

The approach was applied to the FF drive unit of the concept aircraft, considering only a reduced system with the electric motor, the DC/AC inverter and the cabling, while other components are neglected. This study aimed to investigate the influence of a change in DC link voltage level of component as well as system KPIs, especially mass and efficiency. Figure 3 shows the block diagram of the setup.

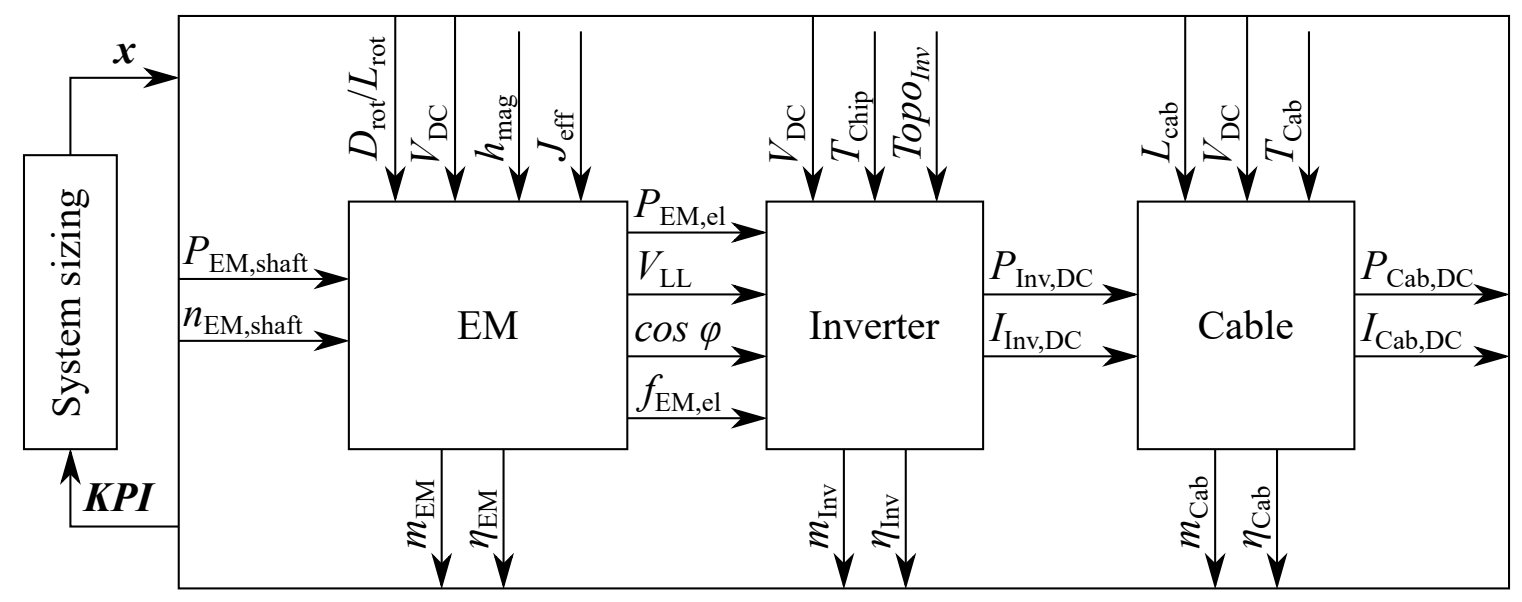

Figure 3. Block diagram of FF drive unit including motor, inverter and cable. For sake of simplicity only one inverter-cable configuration instead of all eight parallel lanes which feed the winding systems of the electric motor is shown.

The diagram only shows the parameters which are relevant for the scope of this study, in detail:

- general requirements (shaft power $P_{E M, \text { shaft }}$, rotational speed $n_{E M, \text { shaft }}$ and diameter-to-lengthratio $D_{\text {rot }} / L_{\text {rot }}$ of the electric machine as well as the cable length $L_{C a b}$ ) which must be fulfilled by the component sizing process;

- global variation parameters which are relevant for more than one component (DC link voltage level $\left.V_{D C}\right) ; ;$

- local variation parameters which only influence the design of one component (conductor current density $J_{\text {eff }}$, inverter topology $T o p o_{\text {Inv }}$, allowable chip $T_{\text {chip }}$ and cable temperature $T_{C a b}$ );

- output/input data, i.e., results from one component sizing process which are necessary for the design process of another component (line-to-line-voltage at the motor terminal $V_{L L}$, power factor $\cos \varphi$, electric frequency $f_{E M, e l}$ and the input DC current of the inverter $\left.I_{I n v, D C}\right)$; and

- $\quad$ key performance indicators (KPI), representing relevant information, such as mass $m$ and efficiency $\eta$ of the components.

General requirements as well as global and local variation parameter define a unique input vector $x$, which results in a unique output vector KPI. The necessity of a fully coupled system sizing model is elucidated using the example of the DC link voltage level. Depending on it, the winding layout as well as insulation aspects in the motor vary, which in term influences the selection of semiconductor 
models during the inverter sizing process. Furthermore, the selection of the DC link voltage level has significant impact on the sizing of the cabling as it is directly proportional to the current. Those effects can only be made visible if the interdependencies between components are considered correctly.

\subsection{Requirements, Constraints and Design Target}

The requirements for the electric motor as well as the semiconductor technology of the inverter and the length of the transmission cable stem from the aircraft design process. The electric motor has eight parallel winding systems each fed by a DC/AC inverter and connected to a DC transmission cable, transferring an eighth of the total power, respectively. The range of the DC link voltage of the transmission system is based on a pre-assessment of available semiconductor blocking voltage levels [27]. As the semiconductor module selection is based on the chosen inverter topology and the DC link voltage, five different topologies including two-, three-, and five-level topologies, are considered. Additionally, several internal parameters for each component are varied within reasonable ranges.

Table 1 summarizes the most important global design requirements as well as global and local variation parameters.

Table 1. Global requirements and input ranges for global and local variation parameters.

\begin{tabular}{|c|c|c|c|c|}
\hline Component & Parameter & Range/Value & Unit & Description \\
\hline \multirow{8}{*}{ Electric machine } & $P_{E M, \text { shaft }}$ & 8000 & $\mathrm{~kW}$ & mechanical shaft power \\
\hline & $n_{E M, \text { shaft }}$ & 2100 & $\min ^{-1}$ & shaft rotational speed \\
\hline & $\tau_{\text {avg }}$ & $6-8$ & $\mathrm{Ncm}^{-2}$ & specific thrust \\
\hline & $D_{\text {rot }} / L_{\text {rot }}$ & $1-2$ & - & diameter-length-ratio rotor \\
\hline & $p$ & $12-50$ & - & number of polepairs \\
\hline & $Q$ & $36-141$ & - & number of stator slots \\
\hline & $h_{m a g}$ & $9-12$ & $\mathrm{~mm}$ & thickness of magnets \\
\hline & $J_{e f f}$ & 17.5 & $\mathrm{~A} \mathrm{~mm}^{-2}$ & conductor current density \\
\hline \multirow{9}{*}{$\mathrm{DC} / \mathrm{AC}$ Inverter } & $P_{E M, e l}$ & 1000 & $\mathrm{kV} \mathrm{A}$ & electric output power \\
\hline & $n_{I n v}$ & 8 & - & number of parallel inverters \\
\hline & & 2L, 3LNPC, & & \\
\hline & Topo Inv & 3LTNPC, & - & topology \\
\hline & & 3LFC, 5LSMC & & \\
\hline & $\operatorname{Tech}_{\text {Inv }}$ & $\mathrm{SiC}$ & - & semiconductor technology \\
\hline & $T_{\text {cool, in }}$ & 75 & ${ }^{\circ} \mathrm{C}$ & cooling inlet temperature \\
\hline & $T_{\text {chip }}$ & 150 & ${ }^{\circ} \mathrm{C}$ & allowable chip temperature \\
\hline & $r_{t h, c a}$ & 105 & $\mathrm{Kmm}^{2} \mathrm{~W}^{-1}$ & $\begin{array}{l}\text { area specific thermal resistance } \\
\text { case to cooling fluid }\end{array}$ \\
\hline \multirow{5}{*}{ DC transmission cable } & $P_{I n v, D C}$ & 1000 & $\mathrm{~kW}$ & DC power per transmission line \\
\hline & $L_{C a b}$ & 86.0 & $\mathrm{~m}$ & total length \\
\hline & $V_{D C}$ & $1500-3000$ & $\mathrm{~V}$ & DC link voltage \\
\hline & $T_{\text {cond }}$ & 120 & ${ }^{\circ} \mathrm{C}$ & allow. conductor temperature \\
\hline & $T_{a m b}$ & 55 & ${ }^{\circ} \mathrm{C}$ & ambient temperature \\
\hline
\end{tabular}

Besides, several constraints, especially concerning the available installation space for motor and inverter, must be considered, as shown in Figure 2. Based on the given input, a large design space exploration is performed. The design target is to identify Pareto-optimal design points with respect to the specified KPIs, i.e., system mass $m_{s y s}$ and efficiency $\eta_{s y s}$, for the given input sets $x$ according to Fang and Qin [28], such that

$$
\min _{x}\left(\left\{m_{s y s}(\boldsymbol{x}),\left(1-\eta_{s y s}(\boldsymbol{x})\right)\right\}\right)
$$

where $m_{s y s}=\sum_{i} m_{i}$ and $\eta_{s y s}=\prod_{i} \eta_{i}$. Pareto-optimality is chosen as criterion to enable an evaluation of designs with concurring KPIs.

Within the next section, the sizing process of each component is explained in more detail. 


\subsection{Detailed Description of Sizing Process of Components}

\subsubsection{Electric Machine}

Several machine types have been studied extensively for electric aircraft [29]. Surface-mounted, permanent magnet synchronous machines (PMSM) have been identified as the most promising technology. PMSM have several DOFs, e.g., the number of poles and slots, the choice of materials for magnets and the laminated core of rotor and stator, the diameter-length-ratio of the rotor $D_{\text {rot }} / L_{\text {rot }}$ or the stator electric current density $J_{e f f}$, which represent the local variation parameters.

The iterative design process of the electric machine includes five disciplines and starts with a defined set of requirements that the final design must fulfil. In the first step, the geometrical layout of the machine is determined. Afterwards, the electromagnetic design includes sizing the magnetic circuit under no-load and load conditions with a non-linear lumped parameter model (e.g., [30,31]), adjusting phase current to reach the required shaft torque $T_{E M, \text { shaft }}$ as well as the configuration of the winding layout. The electromagnetic calculation is done with Simcenter SPEED 13.06 [31]. The structural mechanics section covers the sizing of the retention sleeve for the magnets for different load cases as well as designing rotor shaft and stator housing. The thickness calculation is based on an analytical press-fit model which evaluates the tangential stresses with respect to the radial overclosure of the fit [32,33]. A further important part of machine design is insulation coordination based on IEC 60664 [34] to ensure secure operating voltage levels. To conclude the design process, a thermal analysis of the motor is performed to ensure that waste heat can be dissipated, which especially dimensions the cooling channels. The allowable conductor current density depends on the heat transfer into the cooling medium, which in turn is a function of the thickness of the insulation hindering heat flux and thus decreases allowable conductor current density. Considering the DC link voltage during the motor design thus facilitates a more realistic design.

Passive masses of the machine including rotor shaft, bearings, housing, and terminal box are hard to calculate as they strongly depend on the actual design as well as installation issues. According to a benchmark of several lightweight motors [35], a factor of two was used to account for the passive masses. This is a fair assumption as the power and rotational speed of the machine is fixed and thus the dimensioning torque for all designs identical.

After those design steps, the result is checked against the requirements. If they are not fulfilled the design process enters a further loop, otherwise the design is valid and the specifications are extracted, e.g., to serve as input for a further component such as the DC/AC inverter.

\subsubsection{DC/AC Inverter}

$\mathrm{DC} / \mathrm{AC}$ inverter designs can be realized in different topologies, differing in the height of the single-step output voltage and the total harmonic distortion (THD) $[27,36,37]$. This study only considers two-level (2L-), three-level (T-type) neutral point clamped (3L(T)NCP-) and fly-cap (3LFC-), and five-level stacked multicell (5LSMC-) DC/ AC voltage source inverters. The topologies are explained in detail by the work of Krug [36,37], on which most parts of the design process are based on.

First, the voltage configuration of switches and diodes for the given operation point are determined [36]. A scalable semiconductor characteristic is established from a database with commercially available semiconductor modules and their properties which is then used to perform a coupled calculation of electric and thermal performance. Based on the operation point, the losses of the semiconductor modules are calculated analytically $[36,38,39]$. The semiconductor modules as well as the heat sink, i.e., a cooling plate, are sized such that the chip temperature increase due to the losses at the calculated operating point is below a certain limit and that the waste heat can be removed via the cooling plate at steady-state conditions. As silicon-carbide MOSFETs ( $\mathrm{SiC}$ ) chips are used, a maximum chip temperature of $150^{\circ} \mathrm{C}$ is assumed.

Furthermore, the DC link capacity is calculated based on the expected current ripple calculated analytically using a method provided by Krug [36]. Again, a database of commercially available 
capacitors is used. The sizing process also includes a mass estimation of the gate driver units and a simple housing but misses a calculation of electromagnetic interference (EMI) filters on AC and DC side. To account for the additional mass of the filters, a factor of two is applied.

\subsubsection{Power Transmission Cable}

The power transmission cables are routed from the generators through pylon and wing to the fuselage center to the fuselage aft, where the motor is located. The total length of the cables adds up to $86.0 \mathrm{~m}$. Due to the long distance, aluminum cables are chosen instead of copper cables because of their higher ratio of electrical conductivity to specific weight. A transmission system with eight parallel DC lanes is used to mitigate the risk of a complete power loss in the event of a cable failure [27].

The geometrical design of the DC transmission cable only consists of a circular conductor material, i.e., copper or aluminum, and a concentric layer of insulation material, as electromagnetic field effects and thus shielding can be neglected. The cable sizing process is based on a coupled model of electrical and thermal analysis as well as insulation coordination. The electrical domain covers the calculation of electrical parameters such as voltage levels, currents and resistances as well as the ohmic losses in the conductor considering the conductor material. Insulation coordination determines the necessary thickness of insulation to avoid arcing according to IEC 60664 [34]. A thermal analysis determines the size of the conductor by iteratively solving a one-dimensional, cylindrically symmetrical heat equation [40]. The size of the conductor is chosen such that the losses can be dissipated via natural convection and radiation at given ambient temperature $T_{a m b}$, and the maximum admissible conductor temperature $T_{\text {cond }}$ at the insulation interface is not exceeded.

\section{Results and Discussion of Design Space Exploration}

The results of the design space exploration are discussed for the single components as well as for the combined system.

\subsection{Results for Components}

\subsubsection{Electric Machine}

First, the results of the design space exploration for the electric motor are evaluated. Figure 4 shows the gross mass $m_{E M}$ (active and passive masses) of the electric machine against its electric efficiency $\eta_{E M}$ at full load for three different DC link voltages $V_{D C}$, ranging from $1500 \mathrm{~V}$ to $3000 \mathrm{~V}$. Each marker represents a valid motor design according to the design process described in Section 3.3.1; however, the graph only shows a fraction of the calculated design for better readability.

The Pareto fronts for each voltage level with respect to minimum mass and maximum efficiency (i.e., minimum losses) are depicted as line plots. As can be seen, the higher is the voltage level, the heavier are the machines for identical efficiency levels. This is a result of the increasing thickness of slot, phase, and turn insulation due to the increased voltage level, which in turn leads to a larger necessary slot area. This effect is intensified by the larger cooling channels required due to inferior heat transfer through the thicker insulation and not compensated by lower current levels.

The influence of increased DC link voltage level $V_{D C}$ on the machine efficiency is minor, as especially the copper losses depend on the electric current density of the wire which was unchanged in this study. The slight decrease of efficiency is due to the increased core losses as the absolute loss is proportional to the iron mass at roughly constant specific iron losses. The best machines reach power densities up to $11.0 \mathrm{~kW} \mathrm{~kg}^{-1}$, torque densities up to $50.0 \mathrm{~N} \mathrm{~m} \mathrm{~kg}^{-1}$ and efficiencies up to $98.0 \%$. The peripheral speed of the rotor is moderately high $\left(v_{r o t} \approx 90 \mathrm{~m} \mathrm{~s}^{-1}\right)$ due to the large size $\left(D_{r o t} \approx 0.4 \mathrm{~m}\right)$, wherefore the high power density can be attributed mainly to a high electromagnetic and thermal utilization of materials. This also reflects in a high current density in the stator $\left(J_{e f f}=17.5 \mathrm{~A} \mathrm{~mm}^{-2}\right)$, a value which is challenging but not impossible with state-of-the-art cooling techniques [41]. 


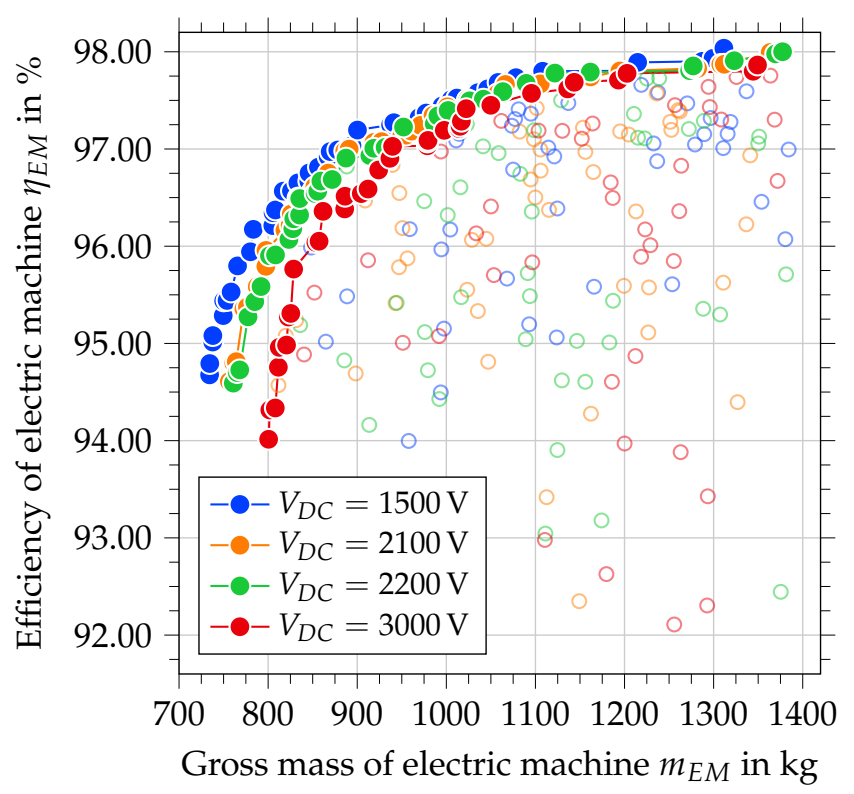

Figure 4. Gross mass against efficiency of electric machine for different DC link voltage levels $V_{D C}$ $\left(P_{E M, \text { shaft }}=8.0 \mathrm{MW}, n_{E M, \text { shaft }}=2100 \mathrm{~min}^{-1}, J_{\text {eff }}=17.5 \mathrm{~A} \mathrm{~mm}^{-2}\right.$ and $\left.f_{E M, e l}=420-1750 \mathrm{~Hz}\right)$. Line plots indicate Pareto-fronts for different voltage levels. Not all results shown for better readability $(\approx 5 \%)$.

\subsubsection{DC/AC Inverter}

As the design of the inverter is based on the use of databases of commercially available semiconductor modules with discrete blocking voltages and capacitors and utilizes different topologies, a different visualization method is chosen. Masses and efficiencies for different topologies are plotted against varying DC link voltage.

Figure 5 shows mass respectively efficiency of several inverter topologies against varying DC link voltage. In Figure 5a, the discrete steps at certain voltages can be seen clearly, which are determined by the allowable utilization accounting for derating at high flight altitudes [27] and the blocking voltage of the semiconductors as well as the number of inverter levels. The maximum efficiency within the different sections which can be reached increases with higher DC link voltages $V_{D C}$ for the different topologies except for the $2 \mathrm{~L}$ inverter due to its inferior loss characteristic at high voltages.

Within the sections, the mass of the inverters decreases slightly due to the lower currents as well as the decreasing current ripple on DC link capacitors and the fly caps. The volatility in mass results from the discrete choice of capacitors out of a database. The absolute higher mass for further increased DC link voltage can be explained by the specifically higher mass of the semiconductor modules with higher blocking voltages (see Figure $5 b$ ).

The most lightweight inverters reach power densities up to $P_{I n v, A C} / m_{I n v} \approx 55 \mathrm{kV} \mathrm{A} \mathrm{kg}^{-1}$ (gravimetric) and $P_{I n v, A C} / V_{I n v} \approx 50 \mathrm{kV} \mathrm{Adm}^{-3}$ (volumetric) at efficiencies of up to $\eta_{\text {Inv }}=99.0 \%$ for maximum chip temperature $T_{\text {chip }}=150^{\circ} \mathrm{C}$ at a fluid inlet temperature $T_{a m b}=75^{\circ} \mathrm{C}$ for a specific resistance $R_{t h, c a}=0.012 \mathrm{KW}^{-1}$ of the heat sink surface to ambient (in this case the liquid). Those values are in good accordance with a literature review [42]. 


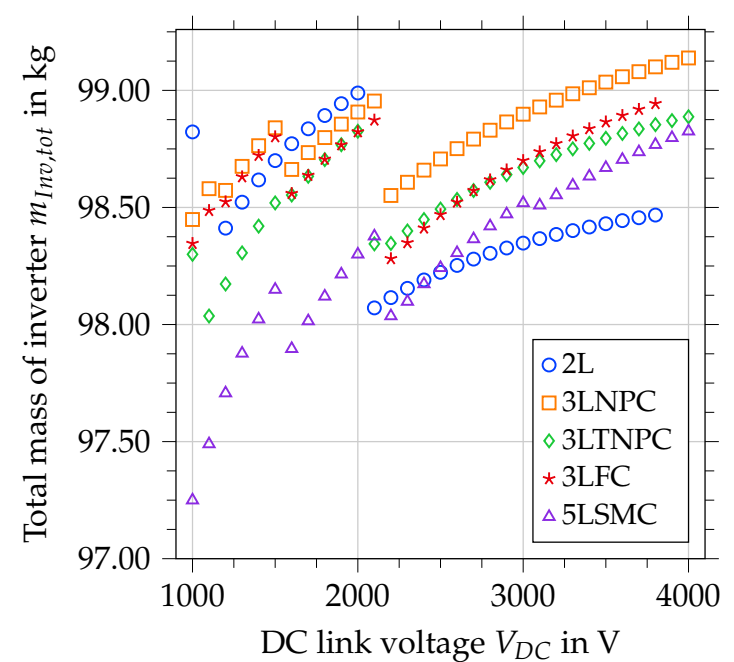

(a) Efficiency $\eta_{I n v}$ vs. DC link voltage $V_{D C}$

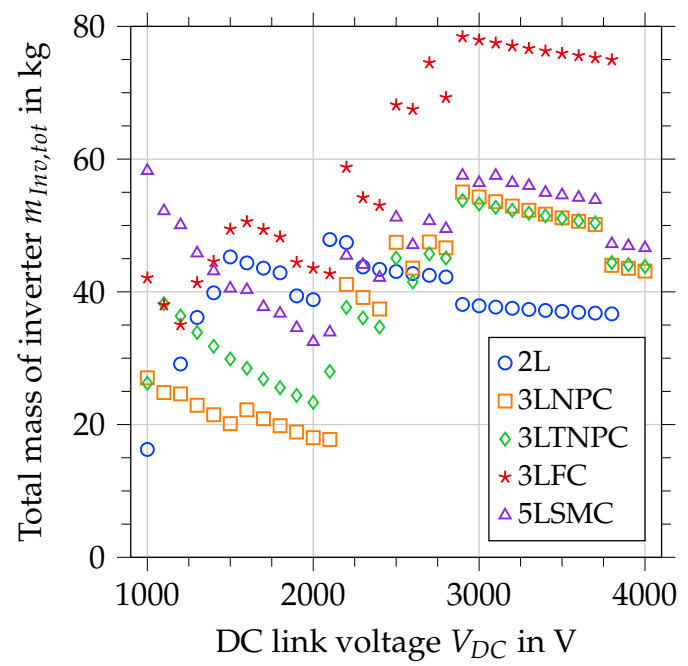

(b) Mass $m_{I n v}$ vs. DC link voltage $V_{D C}$

Figure 5. Efficiency $\eta_{I n v}$ and gross mass $m_{\text {Inv }}$ for varying DC link voltage $V_{D C}$ and different inverter topologies $\left(P_{E M, e l}=1.0 \mathrm{MV} \mathrm{A}, \cos \varphi=0.85, T_{\text {chip }}=150^{\circ} \mathrm{C}, T_{a m b}=75^{\circ} \mathrm{C}\right.$ and $\left.f_{E M, e l}=1000 \mathrm{~Hz}\right)$.

\subsubsection{Transmission Cable}

Figure 6 shows the DC link voltage vs. the cable mass for the eight DC lane configuration specified in Section 3.2 for aluminum as well as copper conductors. While aluminum has only $60 \%$ of the electrical conductivity compared to copper, its density is roughly a third, resulting in $50 \%$ of the cable mass per ampere. The mass of the cable reduces significantly with increasing voltage as the current decreases and with that the Ohmic losses, which are the sizing parameter for the conductor cross-section.

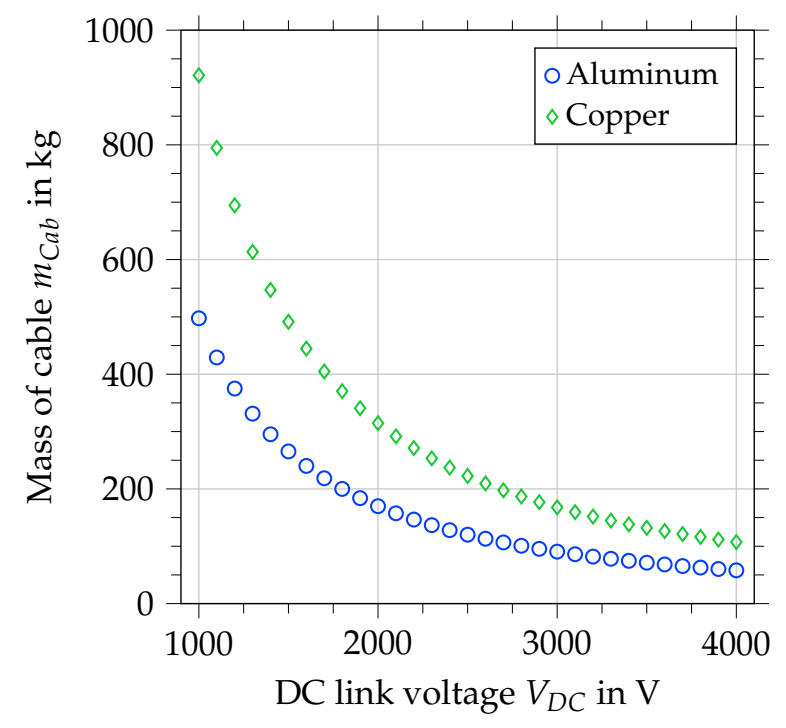

Figure 6. Cable mass $m_{C a b}$ vs. varying DC link voltage $V_{D C}$ of the DC power transmission cable $\left(P_{I n v, D C}=1.0 \mathrm{MW}, T_{C a b}=120^{\circ} \mathrm{C}\right.$ and $\left.L_{C a b}=86.0 \mathrm{~m}\right)$.

With increasing DC link voltage level $V_{D C}$, the cable mass decreases roughly indirectly. For unscreened, stranded aluminum cables with silicone rubber insulation comparable to those in [43], the absolute mass of the insulation only increases slightly for higher voltage levels as the current and thus the conductor cross-section decreases significantly. The relative mass share of the insulation compared to the total cable mass rises from roughly $15 \%$ for $1500 \mathrm{~V}$ to $35 \%$ for $3000 \mathrm{~V}$. 
A high DC link voltage is thus favorable, while a point of minimum cable mass might exist for even higher voltages when insulation starts to dominate the total cable mass.

\subsection{System Results}

Figure 7 shows the results of the CENTRELINE DSE study. Figure 7a outlines the results with respect to the total system mass $m_{S y s}$ and system efficiency $\eta_{S y_{s}}$ of the FF drive unit including the electric motor as well as eight DC/AC inverters and DC power transmission lanes.

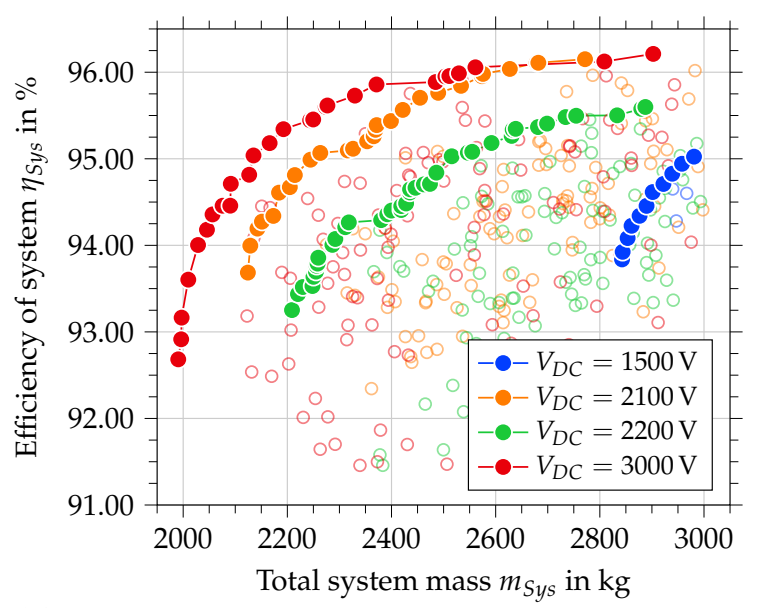

(a) Total system mass $m_{S y s}$ vs. system efficiency $\eta_{S y s}$

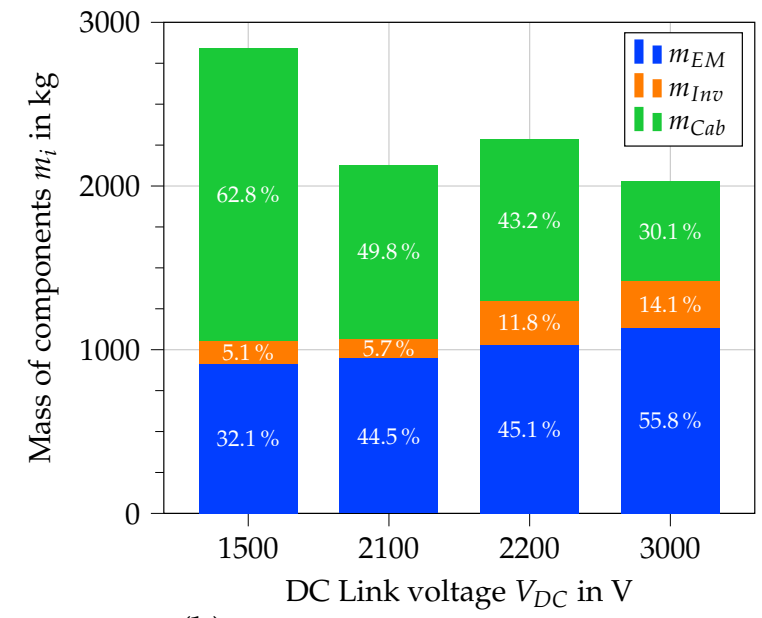

(b) Mass breakdown structure

Figure 7. CENTRELINE DSE study results: (a) Total system mass $m_{S y_{s}}$ against system efficiency $\eta_{S y s}$ of FF drive unit for different DC link voltage levels $V_{D C}$. Line plots indicate Pareto-front for different voltage levels. Not all results shown for better readability $(\approx 5 \%)$. (b) Breakdown of component masses $m_{i}$ including electric machine, inverters, and cables for Pareto-optimal point for different DC link voltages $V_{D C}$ at similar system efficiency $\eta_{S y s} \approx 94.0 \%$.

Two effects superpose each other. First, the general trend implies that, with higher DC link voltage level $V_{D C}$, lower system masses and higher system efficiencies are possible. As both motor and inverter tend to be heavier the mass of the cable must have a major share and its decrease in mass overcompensates the growth of the others. The second effect is more subtle and is explained for the two voltage levels $2100 \mathrm{~V}$ and $2200 \mathrm{~V}$. The first effect would imply that a voltage level of $2200 \mathrm{~V}$ is more suitable on system level; however, this is clearly not the case. While the difference in motor and cable mass for the two voltages is negligible, it is not in terms of inverter efficiency and mass (see Figure 5) as $V_{D C}=2100 \mathrm{~V}$ represents one of the above-mentioned section boundaries where the selected semiconductor blocking voltage and thus the module changes. This results in a quantifiable effect on system level.

To clarify this, Figure $7 \mathrm{~b}$ shows the mass breakdown of the single components for the Pareto optimal points at a similar system efficiency $\eta_{S y s}=94.0 \%$ for the four different voltage levels. Motor and inverter masses only feature a minor rise in absolute terms, while cable masses reduce significantly but still have a significant to dominating share (roughly $30-65 \%$ of the total mass depending on the voltage level).

It can be concluded that a high voltage level is beneficial in terms of system mass, however not for the electric machine and some inverter topologies, without considering further implications such as available semiconductor modules for even higher voltages or increasing complexity. Possible solutions might be the insertion of DC/DC converters after rectifier and before inverter to reduce the voltage level of motor and inverter and increase the one of the DC transmission system or the use of superconducting technologies [11]. This must be evaluated qualitatively. 


\section{Conclusions}

The possibilities of coupled, analytical models for sizing EP systems have been demonstrated for the CENTRELINE project. A sensitivity study on the impact of the DC link voltage level revealed opposing effects on the component properties while on the system level the trade was clearly dominated by the DC power transmission cable. The underlying effect of discrete steps for the blocking voltages of semiconductor modules on the system performance has been made visible. Two potential technical solutions to decrease the influence of the DC power transmission system were given.

Further work will focus on the modeling of other EP components, especially heat exchangers to account for additional masses due to the thermal management needed for an electric aircraft, as well as refining existing models, e.g., passive masses of electric machines and EMI filters of inverters. Optimization strategies will be implemented.

A complete integration of the presented approach by mirroring back the results of the electric propulsion sizing process into the preliminary aircraft design loop would unlock high potential to improve quality of results as well as to optimize them.

Author Contributions: Conceptualization, S.B., M.B. and M.F.; methodology, S.B., M.B. and M.F.; software, S.B., M.B., S.R. and G.W.; formal analysis, S.B.; investigation, S.B.; data curation, S.B.; writing-original draft preparation, S.B.; writing-review and editing, S.B., M.B. and M.F.; visualization, S.B.; supervision, M.B., M.F. and M.N.; project administration, M.B.; and funding acquisition, G.W. and M.B.

Funding: Part of this work was conducted within the CENTRELINE project, which has received funding from the European Union's Horizon 2020 research and innovation programme under Grant Agreement No. 723242.

Acknowledgments: They authors want to thank their colleagues Andreas Mayr, Christoph Marxguth, Mabroor Ahmed and Torsten Schröder for fruitful discussions.

Conflicts of Interest: The authors declare no conflict of interest. The funders had no role in the design of the study; in the collection, analyses, or interpretation of data; in the writing of the manuscript, or in the decision to publish the results.

\section{Abbreviations}

The following abbreviations are used in this manuscript:

EP Electric Propulsion

DOF Degree of Freedom

DSE Design Space Exploration

KPI Key Performance Indicator

DC Direct Current

AC Alternating Current

$\mathrm{CO}_{2} \quad$ Carbon dioxide

$\mathrm{NO}_{x} \quad$ Nitrogen oxide

NASA National Aeronautics and Space Exploration

ICAO International Civil Aviation Organization

ATAG Aviation Transport Action Group

MDAO Multidisciplinary Analysis and Optimization

EU European Union

BLI Boundary Layer Ingestion

GTF Geared Turbo Fan

LPT Low Pressure Turbine

EM Electric Machine

FF Fuselage Fan

SiC Silicon Carbide

PMSM Permanent Magnet Synchronous Machine

THD Total Harmonic Distortion

NPC Neutral Point Clamped 
FC Fly Cap

SMC Stacked Multi Cell

EMI Electromagnetic Interference

\section{References}

1. Penner, J.E.; Lister, D.H.; Griggs, D.J.; Dokken, D.J.; McFarland, M. Aviation and the Global Atmosphere: A Special Report of the Intergovernmental Panel on Climate Change; Cambridge University Press: Cambridge, UK, 1999.

2. Air Transport Action Group. Aviation: Benefit Beyond Borders; ATAG: Geneva, Switzerland, 2018.

3. Follen, G.J.; Del Rosario, R.; Wahls, R.; Madavan, N. NASA's Fundamental Aeronautics Subsonic Fixed Wing Project: Generation N+3 Technology Portfolio; SAE Technical Paper Series; SAE International: Warrendale, PA, USA, 2011.

4. Guynn, M.D.; Berton, J.J.; Tong, M.J.; Haller, W.J. Advanced Single-Aisle Transport Propulsion Design Options Revisited. In Proceedings of the Aviation Technology, Integration, and Operations Conference, Los Angeles, CA, USA, 12-14 August 2013; American Institute of Aeronautics and Astronautics: Reston, VA, USA, 2013.

5. International Civil Aviation Organization. Doc 10127: Independent Expert Integrated Technology Goals Assessment and Review for Engines and Aircraft; ICAO: Montréal, QC, Canada, 2019.

6. International Civil Aviation Organization. Resolution A39-3: Consolidated Statement of Continuing ICAO Policies and Practices Related to Environmental Protection-Global Market-based Measure (MBM) Scheme; ICAO: Montréal, QC, Canada, 2016.

7. Darecki, M.; Edelstenne, C.; Enders, T.; Fernandez, E.; Hartman, P.; Herteman, J.P.; Kerkloh, M.; King, I.; Ky, P.; Mathieu, M.; et al. Flightpath 2050: Europe's Vision for Aviation: Report of the High Level Group; Publications Office of the European Union: Luxembourg, 2011.

8. Aigner, B.; Nollmann, M.; Stumpf, E. Design of a Hybrid Electric Propulsion System within a Preliminary Aircraft Design Software Environment. In Deutscher Luft- und Raumfahrtkongress; Deutsche Gesellschaft für Luft- und Raumfahrt-Lilienthal-Oberth e.V.: Bonn, Germany, 2018; pp. 1-14.

9. Brelje, B.J.; Martins, J.R.R.A. Electric, Hybrid, and Turboelectric Fixed-Wing Aircraft: A Review of Concepts, Models, and Design Approaches. Prog. Aerosp. Sci. 2019, 104, 1-19. [CrossRef]

10. Voskuijl, M.; van Bogaert, J.; Rao, A.G. Analysis and Design of Hybrid Electric Regional Turboprop Aircraft. CEAS Aeronaut. J. 2018, 9, 15-25. [CrossRef]

11. Gemin, P.; Kupiszewski, T.; Radun, A.; Pan, Y.; Lai, R.; Zhang, D.; Wang, R.; Wu, X.; Jiang, Y.; Galioto, S.; et al. Architecture, Voltage, and Components for a Turboelectric Distributed Propulsion Electric Grid (AVC-TeDP); NASA Glenn Research Center: Cleveland, OH, USA, 2015.

12. Gesell, H.; Wolters, F.; Plohr, M. System of Turbo Electric and Hybrid Electric Propulsion Systems on a Regional Aircraft. In Proceedings of the 31st Congress of the International Council of the Aeronautical Sciences (ICAS), Horizonte, Brazil, 9-14 September 2018; pp. 9-14.

13. Pornet, C. Electric Drives for Propulsion System of Transport Aircraft. In New Applications of Electric Drives; Chomat, M., Ed.; InTech: London, UK, 2015.

14. Isikveren, A.T.; Seitz, A.; Vratny, P.C.; Pornet, C.; Plötner, K.O.; Hornung, M. Conceptual Studies of Universally-Electric Systems Architectures Suitable for Transport Aircraft. In Deutscher Luft-und Raumfahrt Kongress; Deutsche Gesellschaft für Luft- und Raumfahrt: Bonn, Germany, 2012.

15. Andrea, J.; Buffo, M.; Guillard, E.; Landfried, R.; Boukadoum, R.; Teste, P. Arcing Fault in Aircraft Distribution Network. In Proceedings of the 2017 IEEE Holm Conference on Electrical Contacts, Denver, CO, USA, 10-13 September 2017; IEEE: Piscataway, NJ, USA, 2017; pp. 317-324.

16. Nya, B.H.; Brombach, J.; Schulz, D. Benefits of Higher Voltage Levels in Aircraft Electrical Power Systems. In Proceedings of the 2012 Electrical Systems for Aircraft, Railway and Ship Propulsion Conference, Bologna, Italy, 16-18 October 2012; pp. 1-5.

17. Jansen, R.; Bowman, C.; Jankovsky, A. Sizing Power Components of an Electrically Driven Tail Cone Thruster and a Range Extender. In Proceedings of the 16th AIAA Aviation Technology, Integration, and Operations Conference, Washington, DC, USA, 13-17 June 2016; p. 3766.

18. Seitz, A. H2020 CENTRELINE-Project Preview. In Proceedings of the 7th EASN International Conference, Warsaw, Poland, 26-29 September 2017. 
19. Pornet, C.; Gologan, C.; Vratny, P.C.; Seitz, A.; Schmitz, O.; Isikveren, A.T.; Hornung, M. Methodology for Sizing and Performance Assessment of Hybrid Energy Aircraft. J. Aircr. 2015, 52, 341-352. [CrossRef]

20. Riboldi, C.E.D.; Gualdoni, F.; Trainelli, L. Preliminary Weight Sizing of Light Pure-Electric and HybridElectric Aircraft. Transp. Res. Procedia 2018, 29, 376-389. [CrossRef]

21. Stückl, S. Methods for the Design and Evaluation of Future Aircraft Concepts Utilizing Electric Propulsion Systems. Ph.D. Thesis, TU München, Munich, Germany, 2016.

22. Vratny, P.C. Conceptual Design Methods of Electric Power Architectures for Hybrid Energy Aircraft. Ph.D. Thesis, TU München, Munich, Germany, 2019.

23. Vratny, P.C.; Hornung, M. Sizing Considerations of an Electric Ducted Fan for Hybrid Energy Aircraft. Transp. Res. Procedia 2018, 29, 410-426. [CrossRef]

24. Isikveren, A.T.; Seitz, A.; Bijewitz, J.; Hornung, M.; Mirzoyan, A.; Isyanov, A.; Godard, J.L.; Stückl, S.; van Toor, J. Recent Advances in Airframe-Propulsion Concepts with Distributed Propulsion. In Proceedings of the 29th Congress of the International Council of the Aeronautical Sciences (ICAS), St. Petersburg, Russia, 7-12 September 2014.

25. Meller, F.; Kocvara, F. Specification of Propulsive Fuselage Aircraft Layout and Design Features: Grant Agreement No. 723242, CENTRELINE Project Deliverable D1.02; Publications Office of the European Union: Luxembourg, 2018.

26. Seitz, A.; Peter, F.; Bijewitz, J.; Habermann, A.; Goraj, Z.; Kowalski, M.; Castillo, A.; Meller, F.; Merkler, R.; Samuelsson, S.; et al. Concept Validation Study for Fuselage Wake-Filling Propulsion Integration. In Proceedings of the 31st Congress of the International Council of the Aeronautical Sciences (ICAS), Belo Horizonte, Brazil, 9-14 September 2018.

27. Wortmann, G. Electric Machinery Preliminary Design Report: Grant Agreement No. 723242, CENTRELINE Project Deliverable D4.04; Publications Office of the European Union: Luxembourg, 2018.

28. Fang, L.C.; Qin, S.Y. Concurrent Optimization for Parameters of Powertrain and Control System of Hybrid Electric Vehicle Based on Multi-Objective Genetic Algorithms. In Proceedings of the 2006 SICE-ICASE International Joint Conference, Busan, Korea, 18-21 October 2006; pp. 2424-2429.

29. Cao, W.; Mecrow, B.C.; Atkinson, G.J.; Bennett, J.W.; Atkinson, D.J. Overview of Electric Motor Technologies Used for More Electric Aircraft (MEA). IEEE Trans. Ind. Electron. 2012, 59, 3523-3531.

30. Hsieh, M.; Hsu, Y. A Generalized Magnetic Circuit Modeling Approach for Design of Surface PermanentMagnet Machines. IEEE Trans. Ind. Electron. 2012, 59, 779-792. [CrossRef]

31. Siemens PLM Software Inc. Simcenter SPEED: PC-BDC 13.06 User's Manual; Siemens PLM Software Inc.: Plano, TX, USA, 2018.

32. Boresi, A.P.; Schmidt, R.J. Advanced Mechanics of Materials, 6th ed.; Wiley: New York, NY, USA, 2003.

33. Eslami, M.R. Theory of Elasticity and Thermal Stresses: Explanations, Problems and Solutions. In Solid Mechanics and Its Applications; Springer: Dordrecht, The Netherlands, 2013; Volume 197.

34. IEC 60664-1:2007. Insulation Coordination for Equipment within Low-Voltage Systems; VDE-Verlag: Berlin, Germany, 2007.

35. Golovanov, D.; Papini, L.; Gerada, D.; Xu, Z.; Gerada, C. Multidomain optimization of High-Power-Density PM Electrical Machines for System Architecture Selection. IEEE Trans. Ind. Electron. 2018, 65, 5302-5312. [CrossRef]

36. Krug, D. Vergleichende Untersuchungen von Mehrpunkt-Schaltungstopologien mit zentralem GleichSpannungszwischenkreis für Mittelspannungsanwendungen. (German) [Comparison of Medium-Voltage Multilevel Converters with Central DC Link, Chap. 3]. Ph.D. Thesis, TU Dresden, Dresden, Germany, 2016.

37. Krug, D.; Bernet, S.; Fazel, S.S.; Jalili, K.; Malinowski, M. Comparison of 2.3-kV Medium-Voltage Multilevel Converters for Industrial Medium-Voltage Drives. IEEE Trans. Ind. Electron. 2007, 54, 2979-2992. [CrossRef]

38. Brückner, T. The Active NPC Converter for Medium Voltage Drives. Ph.D. Thesis, RWTH Aachen, Aachen, Germany, 2006.

39. Wintrich, A.; Nicolai, U.; Tursky, W.; Reimann, T. Application Manual Power Semiconductors; ISLE Verlag: Ilmenau, Germany, 2011.

40. Ilgevicius, A. Analytical and Numerical Analysis and Simulaiton of Heat Transfer in Electrical Conductors and Fuses. Ph.D. Thesis, Universität der Bundeswehr München, Neubiberg, Germany, 2014. 
41. Van der Geest, M.; Polinder, H.; Ferreira, J.A.; Christmann, M. Power Density Limits and Design Trends of High-Speed Permanent Magnet Synchronous Machines. IEEE Trans. Transp. Electrif. 2015, 1, $266-276$. [CrossRef]

42. Kolar, J.W.; Drofenik, U.; Biela, J.; Heldwein, M.L.; Ertl, H.; Friedli, T.; Round, S.D. PWM Converter Power Density Barriers. In Proceedings of the Power Conversion Conference, Nagoya, Japan, 2-5 April 2007; pp. 9-29.

43. Coroplast GmbH \& Co. KG. Wires and Cables for Automotive Applications; Coroplast GmbH \& Co. KG: Wuppertal, Germany, 2016.

(C) 2019 by the authors. Licensee MDPI, Basel, Switzerland. This article is an open access article distributed under the terms and conditions of the Creative Commons Attribution (CC BY) license (http:/ / creativecommons.org/licenses/by/4.0/). 